\title{
A EVOLUÇÃO HISTÓRICA NAS TÉCNICAS DE RINOPLASTIA
}

\author{
HISTORICAL EVOLUTION IN RHINOPLASTY TECHNIQUES
}

Autores:

Júlia Fazoli de Carvalho', Júlia Andrade Bicudo', Rogério Muylaert de Carvalho Britto', Polyana de Paula Mendes Machado', Sara Chagas Abreu'

1 Acadêmico do curso de graduação em medicina da Faculdade de Medicina de Campos (FMC)

Júlia Fazoli de Carvalho; Rua Barão de Miracema, 110, apto 203, Centro, Campos dos Goytacazes - RJ;

(22)997207419; juju.fazoli15@gmail.com - Campos dos Goytacazes / RJ 2020

\section{RESUMO}

A remodelagem nasal, também conhecida como rinoplastia, é um dos procedimentos mais popularmente realizados na cirurgia plástica. Promove a correção da aparência desproporcional do nariz, alterar o tamanho ou formato do nariz, a largura das narinas ou o ângulo entre o nariz e o lábio superior. Além disso, os procedimentos de remodelagem nasal podem ser realizados para corrigir defeitos congênitos e problemas respiratórios crônicos. Existem diferentes técnicas de rinoplastia que sofreram evoluções históricas consideráveis e se adaptam aos fatores individuais de cada paciente.

Palavras chave: rinoplastia, técnicas de rinoplastia, rinoplastia aberta, rinoplastia fechada, rinoplastia em alça de balde.

\begin{abstract}
The nasal remodeling, also known as rhinoplasty, is one of the most popular procedures performed in plastic surgery. It promotes the correction of the disproportionate appearance of the nose, changing the size or shape of the nose, the width of the nostrils or the angle between the nose and the upper lip. In addition, nasal remodeling procedures can be performed to correct birth defects and chronic respiratory problems. There are different rhinoplasty techniques that have undergone considerable historical developments and are adapted to the individual factors of each patient.

Key words: rhinoplasty, rhinoplasty techniques, open rhinoplasty, closed rhinoplasty, delivery rhinoplasty.
\end{abstract}




\section{INTRODUÇÃO}

A rinoplastia foi inicialmente desenvolvido por Sushruta Samhita, na antiga Índia cerca de 500 A.C. em seu texto sobre as rinoplastias para reconstruir narizes que foram amputados como uma punição para os crimes, através de um retalho frontal. A primeira rinoplastia intranasal no Ocidente foi atribuída à Roe em 1887, que foi posteriormente utilizada para fins estéticos por Joseph em 1898.

A cirurgia plástica tem apresentado uma grande procura e a mesma vem aprimorando suas técnicas. É uma área de grande atuação, havendo necessidade da integração de uma equipe multidisciplinar, e dessa maneira alcançar melhores resultados. (SILVA et al., 2004)

A cirurgia de rinoplastia tem a finalidade de remover o excesso, corrigir desvios e assimetrias do nariz, moldando os tecidos remanescentes de modo a formarem um todo harmônico com o conjunto facial (HUNGRIA et al., 2000).

$O$ relatório mais recente da International Society of Aesthetic Plastic Surgery (ISAPS) estima que, em 2017, foram feitas 4.189 .830 intervenções estéticas na face e cabeça. Entre elas, a rinoplastia foi o segundo procedimento mais realizado no mundo, atrás da blefaroplastia.

O Brasil é o país onde a rinoplastia é mais realizada. Em seguida, vêm o México, Estados Unidos, Japão, Itália, Colômbia e Tailândia. No Brasil, das 511.390 cirurgias realizadas na face e na cabeça, a rinoplastia (com 74.890 casos) ficou em terceiro lugar. A rinoplastia foi o procedimento cirúrgico que mais teve aumento (11\%) em todo o mundo. Isso, considerando as técnicas realizadas na face e na cabeça e comparando os dados de 2017 e 2016.

Existem três técnicas de rinoplastia: a rinoplastia aberta, rinoplastia "alça de balde" ou delivery e a rinoplastia fechada.

\section{OBJETIVOS}

O presente trabalho tem por objetivo explorar algumas das técnicas cirúrgicas aplicadas na rinoplastia a fim de elucidar cada um de seus tipos e como são realizadas, mostrando uma evolução histórica das técnicas até as utilizadas nos dias atuais.

\section{RINOPLASTIA ABERTA}

A técnica aberta de rinoplastia foi inicialmente publicada em 1930, evoluindo de forma gradual até os dias atuais. O procedimento consiste na realização de incisões marginais de ambos os lados do nariz, direito e esquerdo, além de uma incisão columelar que as conectam, permitindo a elevação do envelope de pele. A incisão na columela nasal se localiza na porção média, com um "V" invertido, continuando-se com incisões marginais bilaterais. (PIZARRO et al., 2002)

Após as incisões e elevação da pele local, fazse a dissecção retirando ligações fibrosas remanescentes. O periósteo dos ossos nasais na porção caudal é cortado e elevado como parte da cobertura do envelope de partes moles. São então realizadas as correções necessárias para as deformidades nasais, como a septoplastia.

O fechamento é iniciado na incisão columelar, seguido pelo fechamento das incisuras bilaterais. A reforma do contorno nasal se sobrepõe à redução propriamente dita do volume nasal, com uma boa exposição permitindo modificações estruturais precisas. (PIZARRO et al., 2002)

Esse subtipo de rinoplastia possui indicações específicas como traumas anteriores nasais, rinoplastia secundária, malformações congênitas como fenda labial, valvuloplastia nasal, ausência de projeção na cartilagem nasal caudal ou laterorrinia. É a técnica mais empregada para fins acadêmicos e de aprendizagem, pois a via externa permite melhor visualização aos cirurgiões. Diferentemente do método fechado, em que o procedimento é basicamente conduzido pelas sensações táteis, a cirurgia aberta permite a concepção direta, demonstração dos passos e avaliação dos efeitos.

Existem, porém, algumas desvantagens como maior tempo cirúrgico, edema adicional de ponta nasal e cicatriz em região columelar, embora essa se torne imperceptível na grande maioria dos casos. Como complicações, pode haver desenvolvimento de necrose na área columelar, além de hematomas e infecções.

\section{RINOPLASTIA EM ALÇA DE BALDE}

Chamamos de técnica em "alça de balde" ou "delivery" a rinoplastia em que se realiza duas incisões paralelas, uma semelhante à da rinoplastia aberta (na margem das cartilagens) e outra semelhante à da rinoplastia fechada (acima da margem das cartilagens). (MORENO et al., 2020)

A técnica em questão permite que as cartilagens alares sejam expostas adequadamente para que seja possível um remodelamento 
satisfatório que se assemelhe ao máximo à técnica aberta. (MORENO et al., 2020)

A "alça de balde" elimina a cicatriz da columela deixada pela rinoplastia aberta e permite maiores manobras de remodelamento que a rinoplastia fechada, além de permitir luxação das cartilagens alares. (FERNANDES et al., 2018)

Esta prática, dependendo da habilidade do profissional que a aplica, podem gerar resultados igualmente ideais à das outras técnicas. (FERNANDES et al., 2018)

\section{RINOPLASTIA FECHADA}

Na era moderna da rinoplastia, a introdução da rinoplastia aberta ou externa foi saudada por defensores, mas também encontrou forte oposição. Com o tempo, no entanto, o teor deste debate tornar-se mais ecumênico. A maioria dos cirurgiões, atualmente, reconhece a ampla utilidade das abordagens tradicional e endonasal, fechada (CAFFERTY et al., 2016).

A abordagem endonasal pode ser, geralmente, a escolha para pacientes que demandem um perfil conservador de redução, causando menos trauma e, consequentemente, resposta inflamatória reduzida. Suas indicações clássicas incluem: ponta nasal assimétrica, deformidade do nariz torto, deformidade do nariz em sela, deformidade de fenda nasal, rinoplastia secundária exigindo enxerto estrutural complexo e septal (CAFFERTY et al., 2016).

Ademais, cabe salientar que a exposição restrita durante a rinoplastia fechada pode ser uma desvantagem, dependendo do caso e das dificuldades impostas ao amplo acesso nasal (CAFFERTY et al., 2016). Entre outras dificuldades estão: campo visual reduzido, necessidade de experiência, pode induzir instabilidade da parede lateral nasal e limita também o seu ensino para os observadores (BERGHAUS et al., 2016).
Em relação ao reparo específico de anomalias congênitas, destacando-se o lábio leporino unilateral, foi possível concluir, por uma revisão sistemática, que ambas as técnicas fechada e aberta alcançaram uma boa simetria das narinas, sem diminuição do crescimento. No entanto, pela falta de evidências concretas, a superioridade sobre os desfechos tardios não pode ser realizada (JAYARAJAN et al.,2018).

Nesse sentido, em outro artigo buscou-se avaliar aspectos psicossociais ligados ao estresse e possíveis implicações na qualidade de vida dos pacientes, de acordo com o tipo de procedimento ao qual foram submetidos. Revelando-se que, de acordo com determinadas escalas, não houve diferenças entre as abordagens, sendo que, possivelmente, a rinoplastia fechada poderia ter menor impacto por ser menos invasiva (KÜTÜK et al., 2018).

\section{CONSIDERAÇÕES FINAIS}

Todas as técnicas apresentadas mostram vantagens e desvantagens. Tendo em vista a rinoplastia aberta a melhor visualização cirúrgica, torna-a apropriada para aprendizado e vantajosa para indicações específicas como rinoplastia secundária.

Em contraponto o subtipo fechado não apresenta a necessidade de cicatrizes como a aberta, contudo se baseia em sensações táteis do cirurgião, dependendo de sua experiencia. Podendo ser a técnica de escolha para reparo conservador.

A cirurgia em alça de balde encontra uma ponderação entre a técnica aberta e fechada, no qual diminui as cicatrizes do subtipo aberto mas permite correções maiores que o subtipo fechado.

Assim, deve ser levado em consideração a experiência do profissional em associação com as manobras cirúrgicas que serão necessárias.

\section{REFERÊNCIAS BIBLIOGRÁFICAS}

SYKES PJ, Santoni-Rugiu P, MAZZOLA RF. Nicolò Manuzzi (1639-1717) and the first report of the Indian Rhinoplasty. J Plast Reconstr Aesthet Surg. 2008 Dec 18.

HUNGRIA, H. Otorrinolaringologia. 8. ed. Rio de Janeiro: Guanabara Koogan, 2000.

U. PIZARRO, Gilberto, M. DEVUONO, Isabela, G. MOYSÉS, Márcia, FUJITA, Reginaldo Rev. Bras. Otorrinolaringol. vol.68 no.3 São Paulo May 2002. Print version ISSN 0034-7299. Acesso em 05 de setembro de 2020.https://doi.org/10.1590/S0034-72992002000300007 
MORENO, Gustavo R.. Rinoplastia, técnicas cirúrgicas e indicações. Página: Arte Cirúrgica / Cirurgia Plástica. Disponível em: https://www.artecirurgica.com/rinoplastia-tecnicas-cirurgicas-e-indicacoes/. Acesso em: 21 set. 2020.

FERNANDES, Julio Wilson. Anatomia artística e opções técnicas na rinosseptoplastia. Revista Brasileira de Cirurgia Plástica (Rbcp) - Brazilian Journal Of Plastic Sugery, [S.L.], v. 33, p. 40-42, 2018.

GÖKÇE KÜTÜK S, Arıkan OK. Evaluation of the effects of open and closed rhinoplasty on the psychosocial stress level and quality of life of rhinoplasty patients. J Plast Reconstr Aesthet Surg. 2019;72(8):1347-1354.

doi:10.1016/j.bjps.2019.03.020

CAFFERTY A, Becker DG. Open and Closed Rhinoplasty. Clin Plast Surg. 2016;43(1):17-27.

doi:10.1016/j.cps.2015.09.002

BERGHAUS A. Modern Rhinoplasty: Is There a Place for the Closed Approach?. Facial Plast Surg.

2016;32(4):402-408. doi:10.1055/s-0036-1585422 